\title{
3D seismic response of a limited valley via BEM using 2.5D analytical Green's functions for an infinite free-rigid layer
}

\author{
Julieta António*, António Tadeu \\ Department of Civil Engineering, University of Coimbra, Polo II-Pinhal de Marrocos, P-3030-290 Coimbra, Portugal
}

Accepted 15 June 2002

\begin{abstract}
This paper presents analytical solutions for computing the 3D displacements in a flat solid elastic stratum bounded by a rigid base, when it is subjected to spatially sinusoidal harmonic line loads. These functions are also used as Greens functions in a boundary element method code that simulates the seismic wave propagation in a confined or semi-confined $2 \mathrm{D}$ valley, avoiding the discretization of the free and rigid horizontal boundaries.

The models developed are then used to simulate wave propagation within a rigid stratum and valleys with different dimensions and geometries, when struck by a spatially sinusoidal harmonic vertical line load. Simulations are performed in the frequency domain, for varying spatial wave numbers in the axial direction of the valley. Time results are obtained by means of inverse Fourier transforms, to help understand how the geometry of the valley may affect the variation of the displacement field. (c) 2002 Elsevier Science Ltd. All rights reserved.
\end{abstract}

Keywords: 2.5D analytical Green's functions; Wave propagation; Valley formation

\section{Introduction}

It has long been recognized that site effects may significantly affect the motion generated by seismic waves. The site effects generated by topographical features are among the sources of amplifications and de-amplifications of the seismic signals, which can be important over large frequency domains [1-3]. Different analytical and numerical approaches for modeling wave propagation generated by seismic sources have been proposed over the years.

Analytical and semi-analytical solutions have limited their application to the study of scattering and diffraction of waves by geological inclusions and foundations with regular cross-sections placed in a homogeneous medium [4-8]. Numerical methods, such as finite elements and differences, have been used to compute the response within localized domains, like the case of soil structure interaction [9]. In addition to these methods, other researchers have devised techniques for modeling topographic effects, using representation theorems: the direct boundary element method (BEM) [10], and the indirect boundary element method

\footnotetext{
* Corresponding author. Tel.: +351-239-797196; fax: +351-239797190.

E-mail address: julieta@dec.uc.pt (J. António).
}

(IBEM) [11-14]. Another refinement was the development of combinations of integral representations and discrete wave number expansions of Green's functions $[15,16]$. Discrete methods have also occasionally been used to model large alluvial basins [17]. Finally, hybrid methods, which combine semi-analytical representations (to model the exterior domain of the inhomogeneities), and finite elements (to model its interior domain), have also been used [18]. The increased power of computational resources has made it possible to study the 3D behavior of irregular topographies, such as valleys and canyons. Sánchez Sesma [4] analyzed the response of axisymmetric canyons and valleys when subjected to vertically incident P-waves. Sánchez Sesma et al. [19] also studied the scattering from an axisymmetric alluvial valley provoked by SH-waves. Frankel and Vidale [20] used a finite difference method to study the Santa Clara Valley in San Jose, CA. Frankel [21] applied the same technique to the San Bernardino Valley, illustrating how the synthetics seismograms from 3D simulations are markedly different from those computed using 1D and 2D models. Kim and Papageorgiou [22] employed a discrete wave number boundary element method to compute the responses of a hemispherical canyon for incident $\mathrm{SH}, \mathrm{SV}$ and $\mathrm{P}$ waves in a 3D half-space. Reinoso et al. [23] presented a direct BEM for calculating the $3 \mathrm{D}$ scattering of seismic waves 
from irregular topographies and buried valleys due to the incidence of $\mathrm{P}, \mathrm{S}$ and Rayleigh waves. The model is formulated using isoparametric quadratic boundary elements and it makes use of the 3D fundamental displacement. Kattis et al. [24] used a 3D BEM to analyze the vibration isolation provided by a concrete trench and a row of concrete piles.

Many researchers have addressed the study of resonance frequencies in different geologic formations. Bard and Bouchon [25] presented numerical results on the elastic response of symmetrical and homogeneous sediment-filled deep valleys when subjected to the incidence of $\mathrm{SH}, \mathrm{SV}$ and $\mathrm{P}$ plane waves. Their work shows the existence and importance of $2 \mathrm{D}$ resonance patterns. Again, their work revealed that both the $2 \mathrm{D}$ resonance frequencies and amplifications differ markedly from their 1D model. Zhou and Dravinski [26] used an eigenvalue method, based on an indirect boundary integral formulation, to determine the resonance frequencies and mode shapes of deep sediment valleys.

Some researchers have studied the wave propagation for 2.5D formulations (in which the medium is $2 \mathrm{D}$ and the dynamic source $3 \mathrm{D}$, such as a point load), for which the response can be obtained as a sequence of $2 \mathrm{D}$ solutions for different spatial wave numbers, in the direction in which the geometry does not vary.

Analytical solutions of $2.5 \mathrm{D}$ problems are only known for inclusions with simple geometry, such as a circular cylinder. The solution becomes more complex when the 2D geometry is irregular. Luco et al. [27] computed the 3D seismic response of a cylindrical canyon cut in a layered viscoelastic half-space when subjected to the incidence of $\mathrm{P}$ and $\mathrm{S}$ plane waves using an indirect boundary element formulation. Later, Luco and De Barros [28] applied a similar technique to obtain the response of a layered cylindrical valley. Zhang and Chopra [29,30] used the BEM to compute the impedance matrix for a 3D foundation supported on an infinitely long canyon of uniform cross-section cut in a homogenous halfspace, subjected to $\mathrm{P}, \mathrm{S}$ or Rayleigh waves, discretizing the free surface with linear boundary elements.

Pedersen et al. [31] used the IBEM to compute the 3D seismic response of 2D topographies to plane waves, using the full-space Green's functions for a moving harmonic point force. Luco and De Barros [32,33] computed the 3D harmonic response of a system composed by an infinitely long shell with a circular cross-section, buried in a layered vicoelastic half-space when excited by harmonic plane waves impinging at an oblique angle relative to the axis of the shell. An indirect representation for the field in the exterior half-space is combined with a model of the pipeline based on Donnel shell theory. The integral representation of the layered half-space is based on the use of moving Green's functions [34]. Stamos and Beskos [35] calculated 3D dynamic response of long, lined tunnels of uniform crosssection in a half-space subjected to a plane harmonic waves propagating in an arbitrary direction using the BEM formulation. Their model discretizes the free soil surface of the half-space using the full-space Green's function. Santos et al. [36] computed the 3D scattering field obtained when a 2D smooth topographical deformation is subjected to a dilatational point load placed at some point in the medium, using the BEM to discretize the free surface. Tadeu and Santos [37] used the BEM to evaluate the 3D wave field elicited by monopole sources in the vicinity of a fluid-filled borehole. Pei and Papageorgiou [38] simulated the propagation of surface waves in the Santa Clara valley, CA using a hybrid numerical technique, which combines the boundary integral equation method with the finite element method.

2D analyses have also been performed to predict the seismic response of different geological configurations. Huang and Chiu [39] used a 2D numerical scheme based on the boundary integral equation method to study the effects of a canyon on SH wave ground motion. Davis et al. [40] computed the transverse response of underground cavities embedded within an elastic half-space, when subjected to the incidence of SV waves. Analytical solutions are derived for unlined cavities, with the half-space free surface being approximated by a large curved surface. The unlined cavity solution was extended to derive an approximate model for estimating the dynamic hoop forces and bending moments in lightweight flexible liners, subjected to low-frequency waves whose wavelength was much longer than the pipe diameter.

In other related work, Rassem et al. [41,42] computed the seismic site response of alluvial valleys of limited width, using three different engineering models: 1D, 2D and a frame model approach, which allows the prediction of a nonlinear seismic response. The results from the frame model were compared with those given by the $2 \mathrm{D}$ finite element solution, and a good agreement was found. Guan et al. [43] presented a transient Green function caused by suddenly applied line loads in an isotropic and homogeneous halfspace. Dineva and Manolis [44,45] developed a hybrid integral equation, based on both displacement and hypersingular traction formulations, for the analysis of timeharmonic seismic waves propagating through cracked, multi-layered geological regions with surface topography under plane strain conditions.

The present work computes the 3D seismic response of a $2 \mathrm{D}$ valley limited by a free horizontal surface and three rigid boundaries (one horizontal and the other two inclined). The medium is subjected to a vertical sinusoidal harmonic line load, and the displacement wave field near the surface is evaluated using the BEM. Notice that most of the work reported in the literature assumes the incidence of plane harmonic waves. The horizontal, free and rigid surfaces of the present model are not discretized since the Green's functions used are derived in closed form, which accounts for the required boundary conditions at these interfaces. Thus, only the lateral limiting surfaces need to be discretized.

The Green's functions applied here employ the authors' previous technique for defining analytical solutions for the 
steady state response of a homogeneous half-space, and a system built as a single solid elastic layer bounded by two fluid media, subjected to spatially sinusoidal harmonic line loads $[46,47]$. The solid displacement potentials were defined by the authors [48] when evaluating the Green's functions for a harmonic (steady state) line load with a sinusoidally varying amplitude in the third dimension, in an infinite medium. These are in full agreement with the solution for moving loads given earlier by Pedersen et al. [31] and Papageorgiou and Pei [49]. The potentials are written as a superposition of plane waves, according to the technique used first by Lamb [50] for the 2D case, and then by Bouchon [51] and Kim and Papageorgiou [22] for calculating the three-space dimension field by means of a discrete wave number representation.

The computations are first performed in the frequency domain for different spatial wave numbers in the third dimension. Time responses are then obtained by means of inverse Fourier transforms. Complex frequencies are used to avoid aliasing phenomena [52].

This paper first presents the Green's functions for the steady state response of a homogeneous 3D solid layer, limited by a horizontal free surface and rigid horizontal base, when subjected to a spatially sinusoidal harmonic line load. The derived Green's functions for the layer are then incorporated in a direct BEM model to solve the valley problem, which requires only the discretization of the lateral boundaries. The results are validated by applying a direct BEM, using Green's functions for an unbounded space, which requires the full discretization of all the boundaries. The BEM model is then used for simulation analyses to investigate the $3 \mathrm{D}$ wave propagation alteration within a valley, for differing thickness and extent.

\section{Green's functions}

Consider an elastic layer of infinite extent bounded by a flat free surface and a flat rigid base. This system is assumed to be excited by a spatially sinusoidal harmonic line load along the $\mathrm{z}$ direction, applied at point $\left(x_{0}, y_{0}\right)$ in the $x$-, $y$ - or $z$-directions.

The derivation of the present Green's functions requires the solid displacement potentials, and the corresponding Green's functions, defined by the authors [48], when evaluating the Green's functions for a harmonic (steady state) line load with a sinusoidally varying amplitude in the third dimension (see Appendix A), in an infinite medium (full-space), written as a superposition of plane waves $[46,47]$.

The Green's functions for a free-rigid solid layer, with thickness $h$, can be expressed as the sum of the source terms equal to those in the full-space and the surface terms required to satisfy the boundary conditions at the two surfaces (null normal and tangential stresses on the free surface and null displacements along the rigid base). Next, the potentials associated with the surface terms generated by the free (top) and rigid (bottom) surfaces are given.

Load acting in the direction of the $x$-axis:

$$
\begin{aligned}
& \phi_{0}^{x_{-} \text {top }}=E_{a} \sum_{n=-\infty}^{n=+\infty}\left(\frac{k_{n}}{\nu_{n}} E_{b 0} A_{n}^{x}\right) E_{d}, \quad \psi_{x 0}^{x_{x} \text { top }}=0 \\
& \psi_{y 0}^{x_{\text {_top }}}=E_{a} k_{z} \sum_{n=-\infty}^{n=+\infty}\left(\frac{E_{c 0}}{\gamma_{n}} B_{n}^{x}\right) E_{d}, \\
& \psi_{z 0}^{x_{\text {_top }}}=-E_{a} \sum_{n=-\infty}^{n=+\infty}\left(E_{c 0} C_{n}^{x}\right) E_{d} \\
& \phi_{0}^{x_{-} \text {bottom }}=E_{a} \sum_{n=-\infty}^{n=+\infty}\left(\frac{k_{n}}{\nu_{n}} E_{b 0}^{b} D_{n}^{x}\right) E_{d}, \quad \psi_{x 0}^{x_{0} \text { bottom }}=0, \\
& \psi_{y 0}^{x_{0} \text { bottom }}=E_{a} k_{z} \sum_{n=-\infty}^{n=+\infty}\left(\frac{E_{c 0}^{b}}{\gamma_{n}} E_{n}^{x}\right) E_{d}, \\
& \psi_{z 0}^{x_{0} \text { bottom }}=-E_{a} \sum_{n=-\infty}^{n=+\infty}\left(E_{c 0}^{b} F_{n}^{x}\right) E_{d}
\end{aligned}
$$

Load acting in the direction of the $y$-axis:

$$
\begin{aligned}
& \phi_{0}^{y_{-} \text {top }}=E_{a} \sum_{n=-N}^{n=+N}\left(E_{b 0} A_{n}^{y}\right) E_{d}, \\
& \psi_{x 0}^{y_{\text {_top }}}=E_{a} k_{z} \sum_{n=-N}^{n=+N}\left(\frac{-E_{c 0}}{\gamma_{n}} C_{n}^{y}\right) E_{d}, \quad \psi_{y 0}^{y_{\text {_top }}}=0, \\
& \psi_{z 0}^{y^{y} \text { top }}=E_{a} \sum_{n=-N}^{n=+N}\left(\frac{k_{n}}{\gamma_{n}} E_{c 0} B_{n}^{y}\right) E_{d} \\
& \phi_{0}^{y_{-} \text {bottom }}=E_{a} \sum_{n=-N}^{n=+N}\left(E_{b 0}^{b} D_{n}^{y}\right) E_{d}, \\
& \psi_{x 0}^{y_{\text {_bottom }}}=E_{a} k_{z} \sum_{n=-N}^{n=+N}\left(\frac{-E_{c 0}^{b}}{\gamma_{n}} F_{n}^{y}\right) E_{d}, \quad \psi_{y 0}^{y_{0} \text { bottom }}=0, \\
& \psi_{z 0}^{y_{0} \text { bottom }}=E_{a} \sum_{n=-N}^{n=+N}\left(\frac{k_{n}}{\gamma_{n}} E_{c 0}^{b} E_{n}^{y}\right) E_{d}
\end{aligned}
$$

Load acting in the direction of the $z$-axis:

$$
\begin{aligned}
& \phi_{0}^{z-\text { top }}=E_{a} k_{z} \sum_{n=-N}^{n=+N}\left(\frac{E_{b 0}}{\nu_{n}} A_{n}^{z}\right) E_{d}, \\
& \psi_{x 0}^{z_{-} \text {top }}=E_{a} \sum_{n=-N}^{n=+N}\left(E_{c 0} B_{n}^{z}\right) E_{d}, \\
& \psi_{y 0}^{z-\text { top }}=E_{a} \sum_{n=-N}^{n=+N}\left(\frac{-k_{n}}{\gamma_{n}} E_{c 0} C_{n}^{z}\right) E_{d}, \quad \psi_{z 0}^{z \text { top }}=0
\end{aligned}
$$




$$
\begin{aligned}
& \phi_{0}^{z \_ \text {bottom }}=E_{a} k_{z} \sum_{n=-N}^{n=+N}\left(\frac{E_{b 0}^{b}}{\nu_{n}} D_{n}^{z}\right) E_{d}, \\
& \psi_{x 0}^{z \text { bottom }}=E_{a} \sum_{n=-N}^{n=+N}\left(E_{c 0}^{b} E_{n}^{z}\right) E_{d}, \\
& \psi_{y 0}^{z \_ \text {bottom }}=E_{a} \sum_{n=-N}^{n=+N}\left(\frac{-k_{n}}{\gamma_{n}} E_{c 0}^{b} F_{n}^{z}\right) E_{d}, \quad \psi_{z 0}^{z \text { bottom }}=0
\end{aligned}
$$

In Eqs. (1)-(6) $E_{a}=\left(1 /\left(2 \rho \omega^{2} L_{x}\right)\right), E_{d}=\mathrm{e}^{-\mathrm{i} k_{n}\left(x-x_{0}\right)}, E_{b 0}=$ $\mathrm{e}^{-\mathrm{i} \nu_{n} y}, E_{c 0}=\mathrm{e}^{-\mathrm{i} \gamma_{n} y}, E_{b 0}^{b}=\mathrm{e}^{-\mathrm{i} \nu_{n}|y-h|}, E_{c 0}^{b}=\mathrm{e}^{-\mathrm{i} \gamma_{n}|y-h|}, \nu_{n}=$ $\sqrt{k_{p}^{2}-k_{z}^{2}-k_{n}^{2}}$ with $\left(\operatorname{Im}\left(\nu_{n}\right) \leq 0\right), \gamma_{n}=\sqrt{k_{s}^{2}-k_{z}^{2}-k_{n}^{2}}$ with $\left(\operatorname{Im}\left(\gamma_{n}\right) \leq 0\right), k_{n}=\left(2 \pi / L_{x}\right) n, k_{p}=\omega / \alpha, k_{s}=\omega / \beta, \alpha=$ $\sqrt{(\lambda+2 \mu) / \rho}$ and $\beta=\sqrt{\mu / \rho}$ are the velocities for $\mathrm{P}$ (pressure) waves and $\mathrm{S}$ (shear) waves, respectively, $\lambda$ and $\mu$ are the Lamé constants, $\rho$ the mass density, $k_{z}$ the wave number in $z$ and $\mathrm{i}=\sqrt{-1} . L_{x}$ is the distance between the virtual sources placed along the $x$-direction, used to discretize the above equations. These equations can be approximated by a finite sum of terms $(N)$. The parameters $A_{n}^{m}, B_{n}^{m}, C_{n}^{m}, D_{n}^{m}, E_{n}^{m}$ and $F_{n}^{m}(m=x, y, z)$ are as yet unknown coefficients, to be defined by imposing the appropriate boundary conditions.

A system of six equations is defined for each load by imposing the six stated boundary conditions for each value of $n$. The derivation of these systems of equations is quite straightforward, but, since the details are rather unwieldy, the final systems of equations alone are presented in Appendix B.

Once the unknown amplitude of each potential has been calculated, the Green's functions are expressed by the sum of the source terms and the surface terms originated on both the free and rigid surfaces, leading to the following expressions.

Load acting in the direction of the $x$-axis:

$$
\begin{aligned}
G_{x x}= & G_{x x}^{\mathrm{full}}+E_{a} \sum_{n=-N}^{n=+N} \\
& \times\left[A_{n}^{x} \frac{-\mathrm{i} k_{n}^{2}}{\nu_{n}} E_{b 0}+\left(-\mathrm{i} \gamma_{n} C_{n}^{x}-\frac{\mathrm{i} k_{z}^{2}}{\gamma_{n}} B_{n}^{x}\right) E_{c 0}\right] E_{d} \\
& +E_{a} \sum_{n=-N}^{n=+N} \\
& \times\left[D_{n}^{x} \frac{-\mathrm{i} k_{n}^{2}}{\nu_{n}} E_{b 0}^{b}+\left(-\mathrm{i} \gamma_{n} F_{n}^{x}-\frac{\mathrm{i} k_{z}^{2}}{\gamma_{n}} E_{n}^{x}\right) E_{c 0}^{b}\right] E_{d} \\
G_{y x}= & G_{y x}^{\mathrm{full}}+E_{a} \sum_{n=-N}^{n=+N}\left(-\mathrm{i} k_{n} A_{n}^{x} E_{b 0}+\mathrm{i} k_{n} C_{n}^{x} E_{c 0}\right) E_{d} \\
& +E_{a} \sum_{n=-N}^{n=+N}\left(\mathrm{i} k_{n} D_{n}^{x} E_{b 0}^{b}-\mathrm{i} k_{n} F_{n}^{x} E_{c 0}^{b}\right) E_{d}, \\
G_{z x}= & G_{z x}^{\text {full }}+E_{a} \sum_{n=-N}^{n=+N}\left(\frac{-\mathrm{i} k_{z} k_{n}}{\nu_{n}} A_{n}^{x} E_{b 0}+\frac{\mathrm{i} k_{z} k_{n}}{\gamma_{n}} B_{n}^{x} E_{c 0}\right) E_{d} \\
& +E_{a} \sum_{n=-N}^{n=+N}\left(\frac{-\mathrm{i} k_{z} k_{n}}{\nu_{n}} D_{n}^{x} E_{b 0}^{b}+\frac{\mathrm{i} k_{z} k_{n}}{\gamma_{n}} E_{n}^{x} E_{c 0}^{b}\right) E_{d}
\end{aligned}
$$

Load acting in the direction of the $y$-axis:

$$
\begin{aligned}
G_{x y}= & G_{x y}^{\mathrm{full}}+E_{a} \sum_{n=-N}^{n=+N}\left(-\mathrm{i} A_{n}^{y} k_{n} E_{b 0}+\mathrm{i} B_{n}^{y} k_{n} E_{c 0}\right) E_{d} \\
& +E_{a} \sum_{n=-N}^{n=+N}\left(-\mathrm{i} D_{n}^{y} k_{n} E_{b 0}^{b}+\mathrm{i} E_{n}^{y} k_{n} E_{c 0}^{b}\right) E_{d}, \\
G_{y y}= & G_{y y}^{\text {full }}+E_{a} \sum_{n=-N}^{n=+N} \\
& \times\left[-\mathrm{i} \nu_{n} A_{n}^{y} E_{b 0}+\left(\frac{-\mathrm{i} k_{n}^{2}}{\gamma_{n}} B_{n}^{y}+\frac{-\mathrm{i} k_{z}^{2}}{\gamma_{n}} C_{n}^{y}\right) E_{c 0}\right] E_{d} \\
& +E_{a} \sum_{n=-N}^{n=+N} \\
& \times\left[-\mathrm{i} \nu_{n} D_{n}^{y} E_{b 0}^{b}+\left(\frac{-\mathrm{i} k_{n}^{2}}{\gamma_{n}} E_{n}^{y}+\frac{-\mathrm{i} k_{z}^{2}}{\gamma_{n}} F_{n}^{y}\right) E_{c 0}^{b}\right] E_{d}, \\
& +E_{a} \sum_{n=-N}^{n=+N}\left(-\mathrm{i} D_{n}^{y} k_{z} E_{b 0}^{b}+\mathrm{i} F_{n}^{y} k_{z} E_{c 0}^{b}\right) E_{d} \\
G_{z y}= & G_{z y}^{\text {full }}+E_{a} \sum_{n=-N}^{n=+N}\left(-\mathrm{i} A_{n}^{y} k_{z} E_{b 0}+\mathrm{i} C_{n}^{y} k_{z} E_{c 0}\right) E_{d}
\end{aligned}
$$

Load acting in the direction of the $z$-axis:

$$
\begin{aligned}
G_{x z}= & G_{z x}^{\mathrm{full}}+E_{a} \sum_{n=-N}^{n=+N}\left[\frac{-\mathrm{i} k_{z} k_{n}}{\nu_{n}} E_{b}+\frac{\mathrm{i} k_{z} k_{n}}{\gamma_{n}} E_{c}\right] E_{d}, \\
G_{y z}= & G_{z y}^{\mathrm{full}}+E_{a} \sum_{n=-N}^{n=+N}\left[-\mathrm{i} \operatorname{sgn}\left(y-y_{0}\right) k_{z} E_{b}\right. \\
& \left.+\mathrm{i} \operatorname{sgn}\left(y-y_{0}\right) k_{z} E_{c}\right] E_{d}, \\
G_{z z}= & G_{z z}^{\mathrm{full}}+E_{a} \sum_{n=-N}^{n=+N}\left[\frac{-\mathrm{i} k_{z}^{2}}{\nu_{n}} E_{b}+\left(\frac{-\mathrm{i} k_{n}^{2}}{\gamma_{n}}-\mathrm{i} \gamma_{n}\right) E_{c}\right] E_{d}
\end{aligned}
$$

The expressions for the full space $G_{i j}^{\text {full }}(i, j=x, y, z)$ are given in Appendix A. Notice that, if $k_{z}=0$ is used, the system of equations derived above is reduced to four unknowns, leading to the $2 \mathrm{D}$ response.

\section{Green's functions validation}

The displacements given by the Green's functions presented above are compared with those given by a BEM model, which requires the discretization of the free, and rigid surfaces, and the use of the Green's functions for a full space. The unlimited discretization of the free and rigid surfaces in the BEM model is accomplished by using complex frequencies with a 
(a)
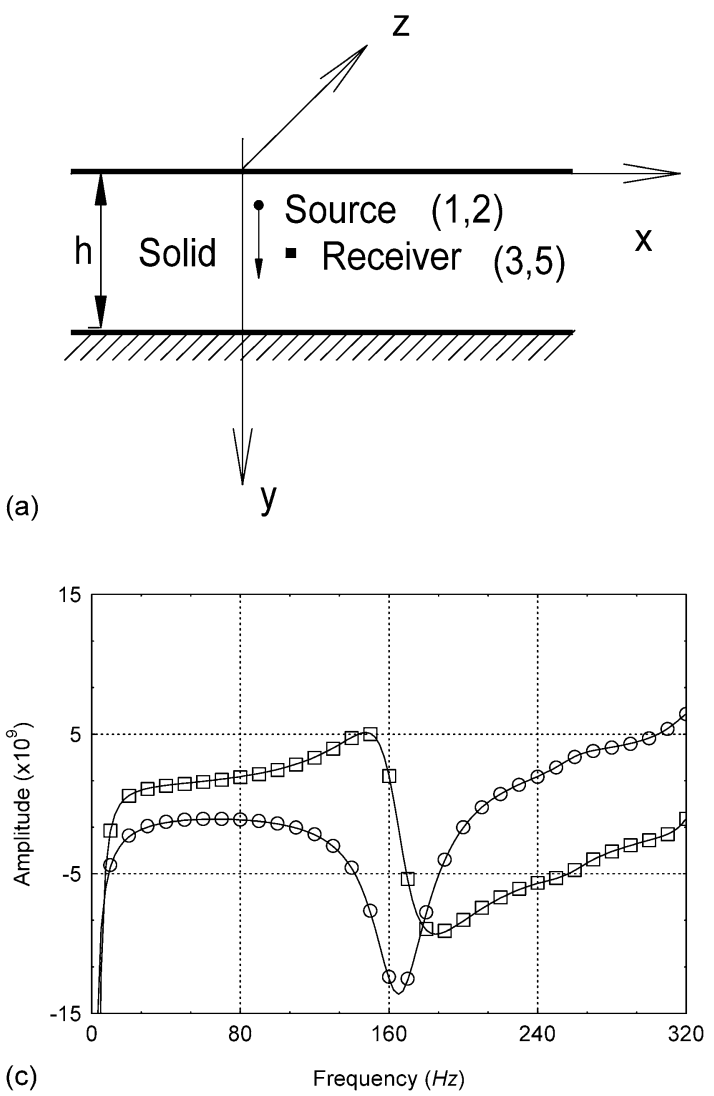
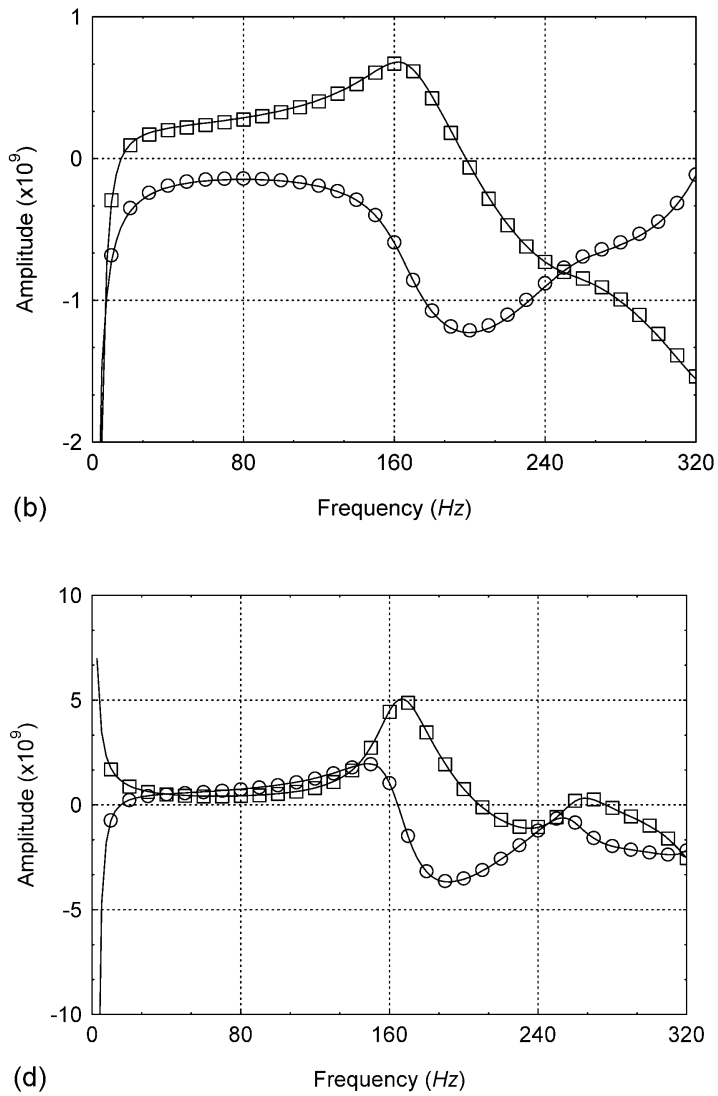

Fig. 1. Spatially sinusoidal harmonic line load along the $z$-direction, acting in the $y$-direction, in a solid formation bounded by a horizontal rigid base: (a) geometry of the problem; (b) $G_{x y}^{\text {surf }}$ solutions; (c) $G_{y y}^{\text {surf }}$ solutions; (d) $G_{z y}^{\text {surf }}$ solutions.

small imaginary part of the form $\omega_{c}=\omega-\mathrm{i} \eta$ (with $\eta=0.7(2 \pi / T))$, which introduces a damping effect $[52$, 53].

A spatially sinusoidal harmonic line load $\left(k_{z}=0.4 \mathrm{rad} /\right.$ $\mathrm{m})$ is applied within a free-rigid solid layer $(\alpha=4208 \mathrm{~m} / \mathrm{s}$, $\beta=2656 \mathrm{~m} / \mathrm{s}$ with $\rho=2140 \mathrm{~kg} / \mathrm{m}^{3}$ ) $10.0 \mathrm{~m}$ thick, at the source point $(x=1.0 \mathrm{~m}, y=2.0 \mathrm{~m})$, acting along the direction $y$. Computations are achieved in the frequency domain $[2.50,320.0 \mathrm{~Hz}]$ with a frequency increment of $2.5 \mathrm{~Hz}$. The surface scattered displacement field in the $i$ direction due to a load acting along $y, G_{i y}^{\text {surf }}$, is computed at a receiver point placed at $x=3.0 \mathrm{~m}$ and $y=5.0 \mathrm{~m}$. The imaginary part of the frequency has been set to $\eta=$ $0.7(2 \pi / T)$, with $T=0.0466 \mathrm{~s}$.

Fig. 1 shows the real and imaginary parts of the displacements. The solid lines represent the analytical responses, while the marked points correspond to the BEM solution. The square marks indicate the real part of the response while the round marks refer to imaginary part.

The two solutions are in very close agreement, but the BEM solution required a very large number of boundary elements, and the use of significant damping. Equally good results were obtained from tests in which different loads and receivers were situated at different points.

\section{Boundary element formulation}

The 3D field generated by a spatially sinusoidal harmonic line load within a valley with a limited width is found by means of the BEM. The present formulation uses the above Green's functions, which take into account the presence of the horizontal free and rigid boundaries. Thus, only the lateral boundaries need to be discretized by boundary elements. A detailed explanation of the BEM equations is not given here since they are widely known [54, 55]. The boundary integral equations, in the absence of distributed loads and in the presence of virtual point loads, $\delta\left(\boldsymbol{x}-\boldsymbol{x}_{0}\right)$, lead to the following equation

$$
\begin{aligned}
c_{i j} u_{j}\left(\boldsymbol{x}_{0}, \omega\right)= & \int_{C} t_{i}(\boldsymbol{x}, \boldsymbol{\nu}, \omega) G_{i j}\left(\boldsymbol{x}, \boldsymbol{x}_{0}, \omega\right) \mathrm{d} s \\
& -\int_{C} H_{i j}\left(\boldsymbol{x}, \boldsymbol{\nu}, \boldsymbol{x}_{0}, \omega\right) u_{j}(\boldsymbol{x}, \omega) \mathrm{d} s
\end{aligned}
$$

where $i, j=1,2$ indicates the normal and tangential directions in relation to the boundary surface; $i, j=3$ represents the $z$-direction; $G_{i j}\left(\boldsymbol{x}, \boldsymbol{x}_{0}, \omega\right), H_{i j}\left(\boldsymbol{x}, \boldsymbol{\nu}, \boldsymbol{x}_{0}, \omega\right)$ are the displacements and tractions in the direction $j$ at $x$, on boundary $C$, originated by a unit sinusoidal line load acting at the source, $\boldsymbol{x}_{0}$, in direction $i$; vector $\boldsymbol{\nu}$ is the unit outward normal at the boundary, and $c_{i j}$ is a constant that equals $\delta_{i j} / 2$ 
for a smooth boundary, where Kronecker's delta is represented by $\delta_{i j}$.

Since the boundaries of the valley to be discretized are the rigid lateral surfaces, which require null displacement boundary conditions, a simplified form of Eq. (10) is obtained

$\int_{C} t_{i}(\boldsymbol{x}, \boldsymbol{\nu}, \omega) G_{i j}\left(\boldsymbol{x}, \boldsymbol{x}_{0}, \omega\right) \mathrm{d} s=0$

Equilibrium relations are used to transform the displacement fields into the normal, tangential and local $(z)$ co-ordinate systems at each element. The boundary and variable values need to be discretized before this integral can be evaluated for an arbitrary cross-section. Therefore, when $N$ boundary elements are used and nodal displacements within each element are taken to be constant, and to have the same value at the respective nodal point, Eq. (11) becomes

$\sum_{n=1}^{N} G_{i j}^{k n} t_{j}^{n}=0$

In Eq. (12), the element number at the point where the virtual load is applied is defined by $k$, the boundary values in element $n$ are identified by $t_{j}^{n}$, and $G_{i j}^{k n}$ represents the element integrals

$G_{i j}^{k n}=\int_{C_{n}} G_{i j}\left(\boldsymbol{x}, \boldsymbol{x}_{0}, \omega\right) \mathrm{d} s$

where $C_{n}$ is the boundary segment.

If a virtual load is applied on the boundary, in each node, a system of linear equations relating nodal forces and nodal displacements is obtained. These equations can be solved for the nodal tractions.

When an incident wave illuminates the valley, Eq. (11) is changed as follows

$\int_{C} t_{i}(\boldsymbol{x}, \boldsymbol{\nu}, \omega) G_{i j}\left(\boldsymbol{x}, \boldsymbol{x}_{0}, \omega\right) \mathrm{d} s+u_{j}^{\text {inc }}\left(\boldsymbol{x}_{0}, \omega\right)=0$

The incident field in this equation is given analytically as the sum of the source terms (2.5D full-space) and the surface terms originated on both free and rigid horizontal surfaces (described in Appendix B). After mathematical manipulation of the integral equations, combined and subjected to the boundary conditions, and discretized appropriately, a system of equations is formulated that can be solved for the nodal tractions.

The required integrations in Eq. (14) are performed using Gaussian quadrature when the element to be integrated is not the loaded element. For the loaded element, the existing singular integrands in the source terms of the Green's functions are calculated in closed form [56,57], while the integrands involving the surface terms originated on both free and rigid surfaces are solved using a Gaussian quadrature scheme.

A numerical inverse fast Fourier transform in the frequency domain allows the displacements to be obtained in the time domain. In the present work, the source is modeled as a Ricker wavelet. The Fourier transformations are achieved by discrete summations over frequencies, which is mathematically the same as adding periodic sources at temporal intervals $T=(2 \pi / \Delta \omega)$, where $\Delta \omega$ represents the frequency steps. Complex frequencies are used, in the form $\omega_{c}=\omega-\mathrm{i} \eta$ (with $\eta=0.7 \Delta \omega$ ), to avoid aliasing phenomena. In the time domain, this effect is removed by rescaling the response using an exponential window $\mathrm{e}^{\eta t}[58]$.

\section{BEM validation}

The present BEM algorithm was implemented and validated by comparing the results with those provided by a BEM code, which requires the full discretization of all valley boundaries. To validate the response, a smaller valley was chosen, as shown in Fig. 2(a), with the same material properties as the free-rigid solid layer. A spatially sinusoidal harmonic line load $\left(k_{z}=0.4 \mathrm{rad} / \mathrm{m}\right)$ is applied at $(x=-4.0 \mathrm{~m}, y=3.0 \mathrm{~m})$, actuating along the direction $y$. Computations are performed in the frequency range [2.50,320.0 Hz] with a frequency increment of $2.5 \mathrm{~Hz}$. The scattered displacement field is computed at a receiver point placed at $x=-6.0 \mathrm{~m}$ and $y=6.0 \mathrm{~m}$. Fig. 2(b) $-(\mathrm{d})$ show the computed results: solid lines represent the new BEM solutions, while the marked points correspond to the BEM solution using the full boundary discretization. The square marks represent the real part of the response while the round marks indicate the imaginary part.

Analysis of the results reveals a very good agreement between the two solutions. However, it should be pointed out that the BEM formulation, using the full boundary discretization, will not be suitable for computation involving large, extended valleys.

\section{Applications}

The analytical and the BEM solutions are used to study the effect of a stratum with a rigid base or a valley on the seismic wave propagation generated by a harmonic sinusoidal line load acting along the vertical direction. The valley is assumed to have only one or two lateral confinements, which can be inclined or vertical. Three different configurations are therefore analyzed: the first assumes that the stratum is infinitely long (Model 1); the second introduces an inclined lateral rigid confinement (Model 2); the third simulates a valley bounded by two inclined lateral rigid surfaces (Model 3) (see Fig. 3). The first model is solved analytically, whereas the second and the third models are simulated using the BEM to obtain the solutions.

The topographical free surface and the horizontal rigid boundary of the various models are not discretized. The 


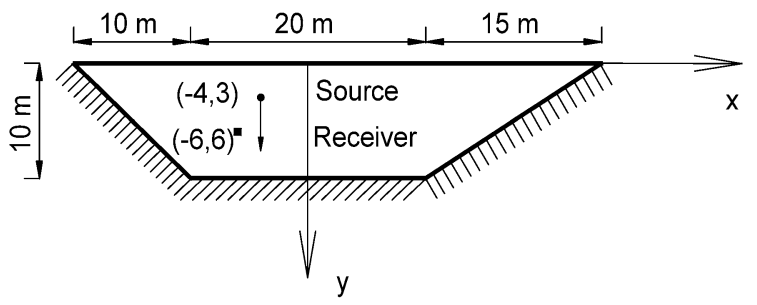

(a)

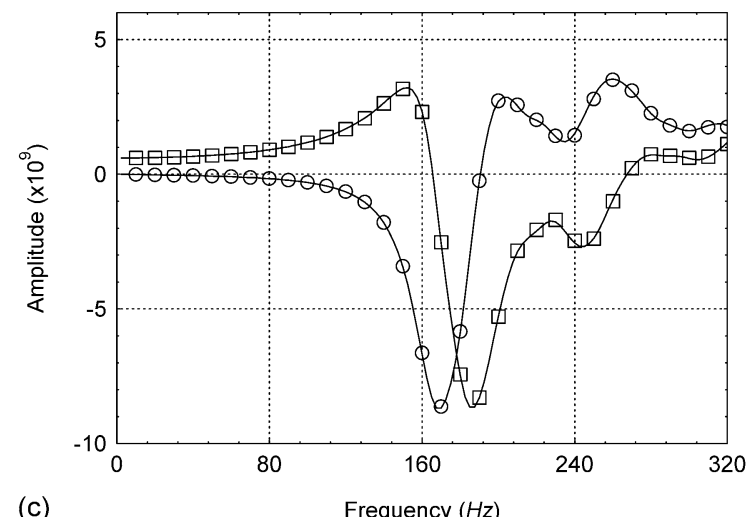

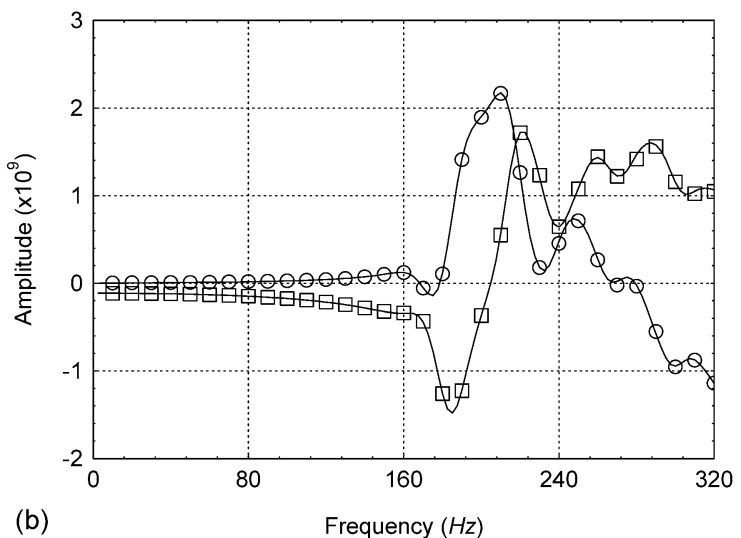



Fig. 2. Spatially sinusoidal harmonic line load along the $z$-direction, acting in the $y$-direction, in a valley formation, applied in the $y$-direction: (a) geometry of the problem; (b) horizontal $(x)$ displacements; (c) vertical $(y)$ displacements; (d) displacements along the $z$-direction.

lateral confinements of the valley are modeled with a number of boundary elements, defined according to the excitation frequency of the harmonic source. The ratio between the wavelength of the incident waves and the

(a)

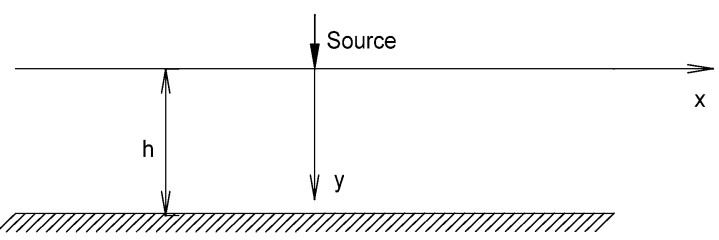

(b)

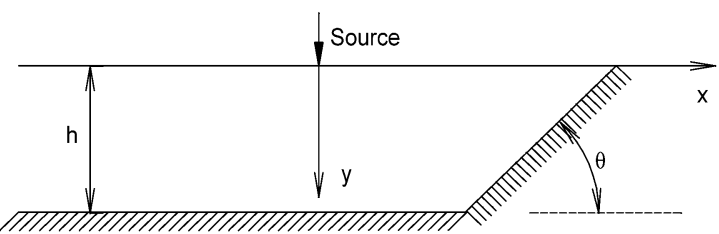

(c)
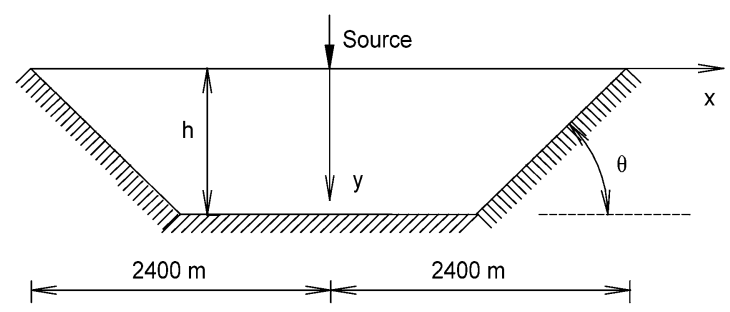

Fig. 3. Geometries of the numerical application models: (a) Model 1; (b) Model 2; (c) Model 3. length of the boundary elements is kept to a minimum of 12 . In no case, however, is the number of the boundary elements used to model each surface less than 20 .

The material properties of the medium remain constant for all analyses and are equal to those used in the validation of the Green's functions and the BEM code $(\beta=2656 \mathrm{~m} / \mathrm{s}$, $\alpha=4208 \mathrm{~m} / \mathrm{s}$ and $\rho=2140 \mathrm{~kg} / \mathrm{m}^{3}$ ).

The displacements produced by the vertical load, applied at $(0.0,2.0 \mathrm{~m})$ are recorded along evenly spaced $(40.0 \mathrm{~m})$ receivers, distributed along one line placed horizontally at $1.0 \mathrm{~m}$ below the free surface.

Responses are computed for different values of $k_{z}$, that is following waves with different apparent wave velocities along the $z$-axis. Selected simulations are presented for three apparent velocities $(c)$ : infinite $c, c=4208 \mathrm{~m} / \mathrm{s}$ and $c=$ $2656 \mathrm{~m} / \mathrm{s}$. Infinite $c$ represents waves arriving at the receivers with a $90^{\circ}$ inclination in relation to the $z$-axis, and corresponds to a pure $2 \mathrm{D}$ problem. As the path inclination decreases from 90 to $0^{\circ}$, there is a lower bound value for $c$ that represents the slowest guided surface waves, which correspond to the Rayleigh waves.

Computations are performed in the frequency range $[0.125,16.0 \mathrm{~Hz}]$, with a frequency increment of $0.125 \mathrm{~Hz}$, which determines the total duration $(T=8.0 \mathrm{~s})$ of the analysis in the time domain. The time solutions are obtained assuming the existence of a source with a Ricker time 
(a)

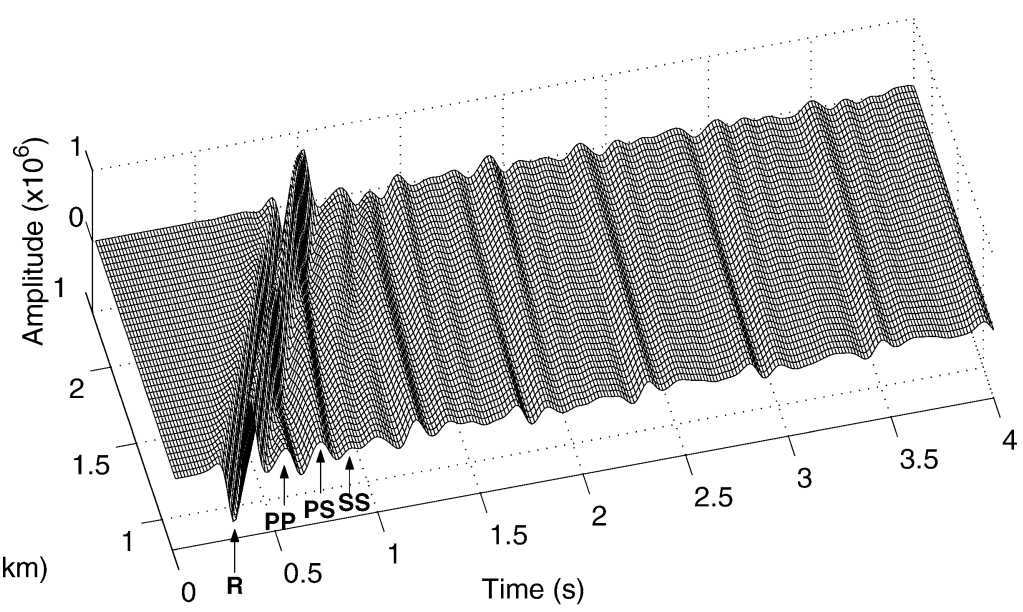

Time (s)

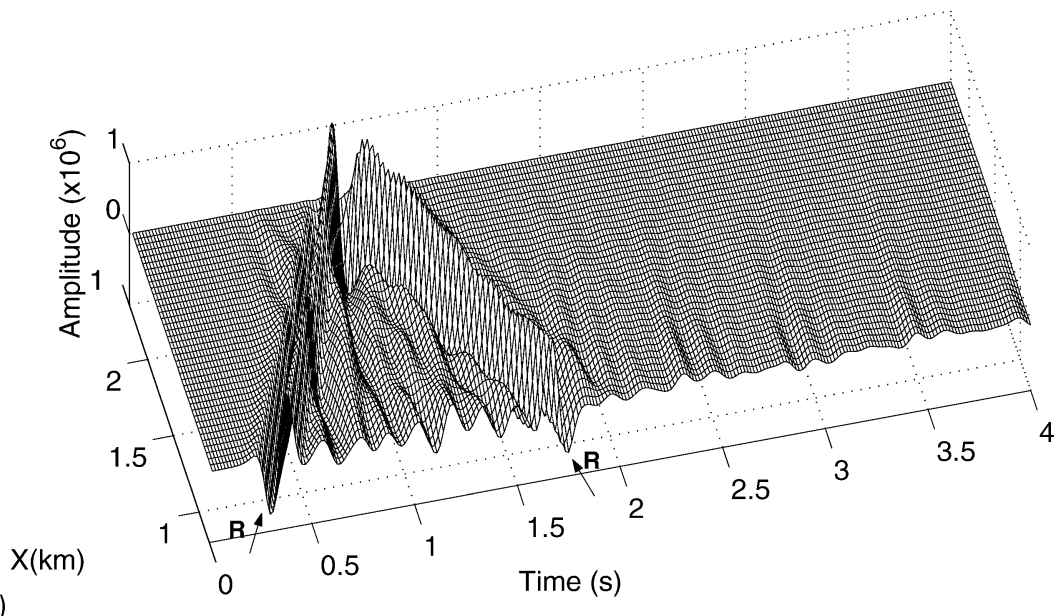

(b)

(c)

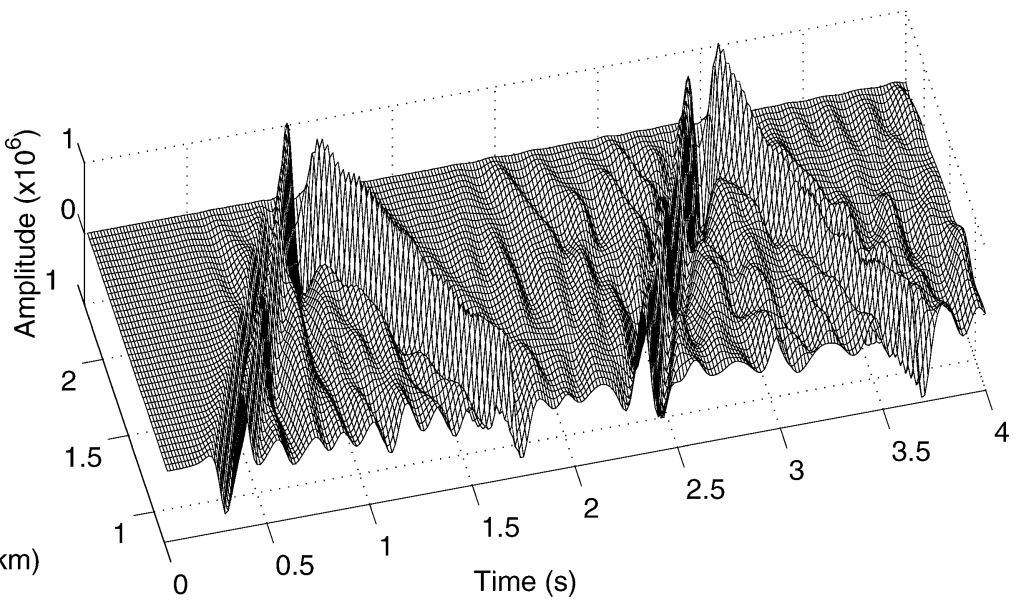

Fig. 4. Vertical displacements when the vertical load $\left(k_{z}=0\right)$ is placed in an elastic formation $1200 \mathrm{~m}$ thick: (a) Model 1; (b) Model $2\left(\theta=45^{\circ}\right)$; (c) Model 3 $\left(\theta=45^{\circ}\right)$.

dependence, with a characteristic frequency of $5.0 \mathrm{~Hz}$. A selection of synthetic seismograms is displayed for receivers placed from 820.0 to $2380.0 \mathrm{~m}$ up to $4.0 \mathrm{~s}$.

Fig. 4 presents synthetic seismograms in the time domain for the vertical $(y)$ displacement when a vertical line load $\left(k_{z}=0\right)$ is placed in a free-rigid elastic formation, $h=$ $1200 \mathrm{~m}$ thick. Fig. 4(a) shows the response obtained when the stratum is of infinite extent (Model 1). At time $t=0$, the load creates a first dilatational and a first shear cylindrical pulse that propagate away from it. The spacing of the wave fronts is in accordance with their differing velocities. A surface pulse follows, which corresponds to the Rayleigh waves. Fig. 4 shows the predominance of these Rayleigh waves (labeled $R$ ). The presence of the first dilatational 
pulse cannot be seen. The horizontal displacement would show a small earlier-arriving pulse related to the dilatational $\mathrm{P}$ wave (not illustrated). Notice that, the $\mathrm{S}$ waves arrive before the Rayleigh waves, but they do not fully separate into two pulses because the difference in velocities is only slight. As time elapses, some of these waves hit the rigid base and a reflected field will be created. Part of this wave field travels back to the free surface where it is subjected to a new scattering phenomenon. This leads to a complex wavefield, caused by multi-wave reflections at the free surface and at the rigid base of the stratum. As the wave pulses travel back and forth between the surface and the floor of the stratum, they lose energy to the surrounding medium, and dissipate. The first set of pulses (labeled $R$ ) are followed by $\mathrm{P}$ waves reflected from the rigid base (labeled $P P$ ), $\mathrm{S}$ mode converted waves resulting from the $\mathrm{P}$ wave incidence on the rigid base (labeled $P S$ ) and $\mathrm{S}$ waves reflected from the incidence of $\mathrm{S}$ waves on the same boundary. The arrival times found for these different pulses are consistent with the predictions given by ray acoustics. It is further observed that the wave front of these train pulses becomes flatter for higher order reflections. With the passage of time, additional surface waves are produced by the incidence of waves on the free surface of the stratum.

Fig. 4(b) displays the synthetic waveforms produced when the second formation configuration is simulated, ascribing an inclination of $45^{\circ}$ to the lateral confinement (Model 2). The comparison of these results with those presented in Fig. 4(a) reveals pronounced differences. The Rayleigh wave strikes the lateral rigid boundary, is reflected back, and travels in the opposite direction. The amplitude of these surface waves is now larger than before, and its enhanced amplitude is observed mainly in the vicinity of the lateral confinement. The largest amplitude is about 50\% larger in relation to the maximum amplitude registered with Model 1. The amplitude of the waves generated by the multi-reflections of the horizontal boundaries decreases, particularly for receivers placed in the vicinity of the lateral confinement. This phenomenon is explained by the nature of the lateral boundary, which ascribes null displacements to its surface, and by the reflective power created by the inclination of the confinement, which enables energy to be reflected away.

Fig. 4(c) gives the responses computed when the valley formation (Model 3) is built with lateral boundaries at an inclination of $45^{\circ}$. Wave features similar to those found in the previous model are registered. Additional Rayleigh wave pulse trains are observable, the result of the multireflections between both lateral confinements. The amplitude of the wavefield increases about $20 \%$ in relation to that registered within the Model 2.

This behavior agrees with the results obtained by Aki [3], and Bard and Bouchon [25], who confirm that the finite lateral extent of surface layers introduces other effects, such as the generation of additional surface waves at the edges and the resonance in a lateral direction, which tends to increase the amplitude as well as the duration of the ground motion.

To better understand the resonance phenomena of the valley, the frequency spectra responses up to $4.0 \mathrm{~Hz}$ are also included for receivers placed from -2380.0 to $2380.0 \mathrm{~m}$. Fig. 5(a) shows the spectrum associated with the vertical displacements obtained in the presence of Model 1. As expected, the response is symmetric in relation to the position of the load. Two well-developed resonance peaks are visible. The first, at $f_{h}=\alpha /(4 h)=0.88 \mathrm{~Hz}$, corresponds to the 1D resonance frequency. The second resonance frequency is spaced from the first $f_{h}=\alpha /(2 h)=$ $1.75 \mathrm{~Hz}$. Fig. 5(b) gives the frequency spectra response obtained when the load is placed within a layer formation bounded by a lateral confinement (Model 2). The extra wave reflections produced by the lateral confinement, observed in the time domain plot, originate a complex resonance effect that is clearly visible at receivers placed between the load and the confinement. On the other side of the load, the peaks observed in Model 1 are still evident. However, additional frequency spectra are present between those resonance peaks. Fig. 5(c) shows the frequency spectra response obtained within the valley formation (Model 3) for the vertical displacements. As expected, given the time responses, this plot exhibits a complex structure pattern. The ground motion amplification is easily discernible. Furthermore, the resonance frequencies associated with both the vertical and lateral interferences are well defined.

Fig. 6 presents the seismic responses when the thickness of the formation is reduced to $400.0 \mathrm{~m}$. To better understand the importance of the inclination of the lateral confinement, an additional geometric formation has been included, which assumes the existence of a vertical lateral confinement $\left(\theta=90^{\circ}\right)$. The amplitude of the responses increases when compared with those obtained for a formation $1200.0 \mathrm{~m}$ thick (about 15\%). The responses of the Model 1 reveal the existence of multiguided waves, which becomes more visible as the distance from the receiver to the source increases (see Fig. 6(a)). The vertical displacements generated by Model 2, where $\theta=45^{\circ}$, again demonstrate the decay of amplitude for receivers in the vicinity of the lateral confinement, particularly after the arrival of the first guided waves (see Fig. 6(b)). The wavefield produced away from the lateral confinement is enhanced by the reflective power of the lateral boundary. Fig. 6(c) shows the computed response when the lateral confinement is vertical. It may be seen that although the field in the immediate vicinity of the lateral boundary is reduced, it is increased in comparison with the fields obtained for an inclined confinement. The results for Model 3 follow the features described above, but exhibit pulses reflected from the second lateral wall.

Fig. 7 shows the vertical displacements at receivers placed in the Model 3, with a formation $400.0 \mathrm{~m}$ thick and $\theta=45^{\circ}$, when the apparent velocity is $c=4208$ and 

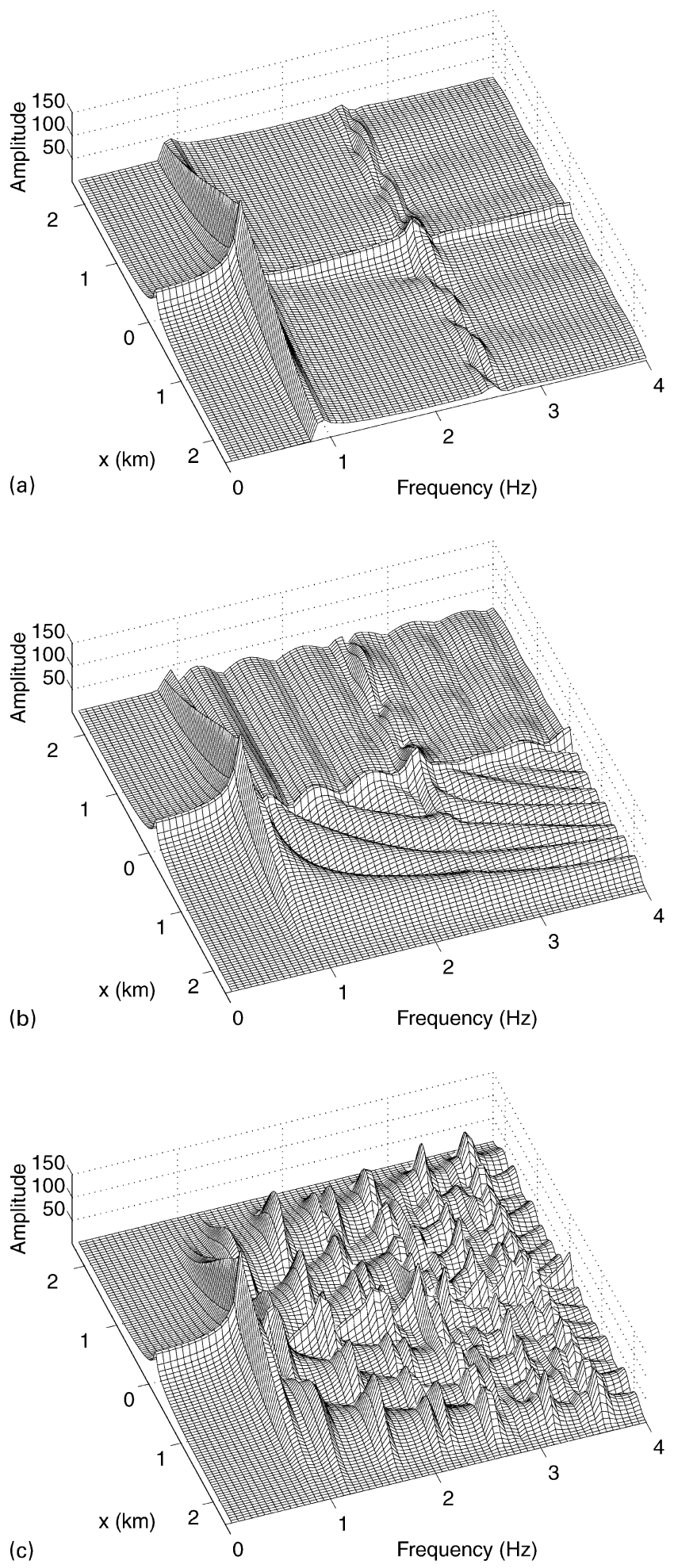

Fig. 5. Frequency spectra responses associated with the vertical displacements when the vertical load $\left(k_{z}=0\right)$ is placed in an elastic formation 1200 m thick: (a) Model 1; (b) Model $2\left(\theta=45^{\circ}\right)$; (c) Model $3\left(\theta=45^{\circ}\right)$.

$2656 \mathrm{~m} / \mathrm{s}$. As the apparent velocity decreases, the arrival times of the different pulses and their amplitude also decrease, indicating that the scattering energy is mainly concentrated in the vertical z-plane containing the dynamic source. The amplitude decreases by about 15 and $20 \%$ in relation to $c=\infty \mathrm{m} / \mathrm{s}\left(k_{z}=0\right)$ (Fig. 6(d)) when $c=4208$ and $2656 \mathrm{~m} / \mathrm{s}$, respectively.

A pulse in these plots, with a travel time $\tau$, corresponds to waves that travel from the source to the reflector and then to the receiver, along the same ray path inclination in relation to the $z$-axis. The travel distance $(L)$ in this domain is smaller because it corresponds to the projection of the initial vertical path $(d)$ relative to the inclined path, giving a distance $L=d \sin [\arccos (v / c)]$. A fall in the apparent velocity thus causes a better separation of the $\mathrm{P}, \mathrm{S}$ and Rayleigh waves. When the apparent velocity equals the velocity of the $\mathrm{P}$ waves (see Fig. 7(a)), the waves traveling at this velocity arrive at the various receivers at $t=0.0 \mathrm{~s}$, and only the waves traveling at lower velocity, the $S$ and Rayleigh waves, survive in these time plots. Furthermore, when the apparent velocity is assumed to be $c=2656 \mathrm{~m} / \mathrm{s}$, only the guided waves (Rayleigh waves) survive (see Fig. $7(b))$.

\section{Conclusions}

The analytical functions developed and used to obtain the 3D displacement field in a solid stratum with a rigid base were both interesting in themselves and useful when incorporated as Greens functions in a BEM code to compute the wave field in a 2D valley formation. These functions and the BEM model were found to be efficient and helpful in the context of seismic research.

Synthetic seismograms were built, following waves with different apparent wave velocities along the $z$-axis of the 2D valley. The responses obtained for a free-rigid elastic formation exhibit a set of periodical pulses in the time domain, caused by the multi-reflections between the free surface and the rigid base. Initially, the wave fronts associated with the body waves propagating away from the source are circular, but as time develops they get flatter.

When a lateral inclined rigid boundary is introduced, the original guided waves are reflected back after impinging on this confinement. It produces the enhancement of the former guided waves' amplitude, particularly in the vicinity of the confinement. However, the amplitude of the pulses originated by the multi-reflections in the horizontal boundaries decreases, more noticeably at receivers in the vicinity of the lateral boundary. This fall in amplitude is due to the null displacements imposed by its boundary conditions and to its large reflective power. When the lateral boundary is vertical, the amplitude of the wavefield increases in the immediate vicinity of the lateral confinement, owing to the diminished reflecting power of the boundary.

When the valley formation is built with two lateral boundaries, additional Rayleigh pulse reflections occur, leading to the enhancement of the displacement field amplitude at receivers in the vicinity of the lateral boundary. 
(a)

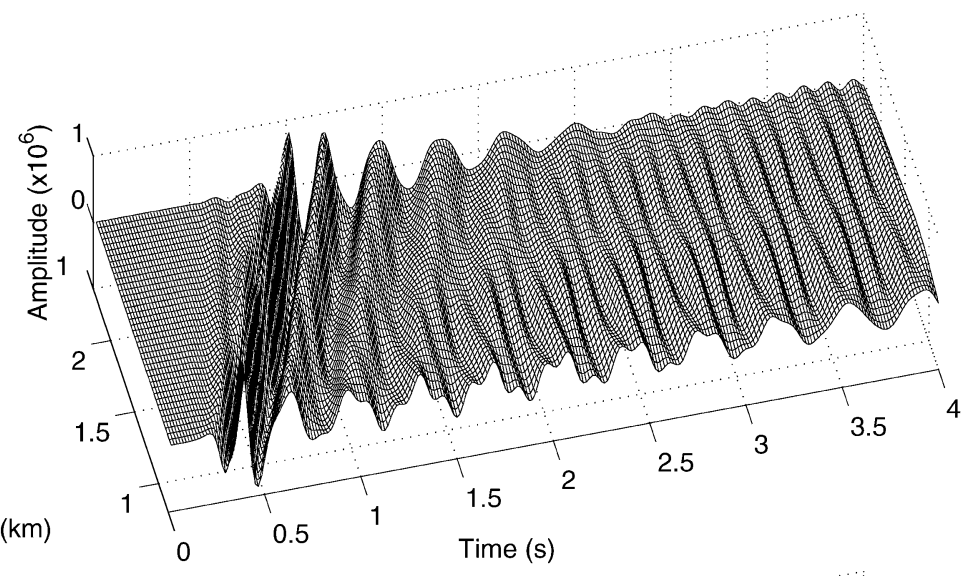

(b)

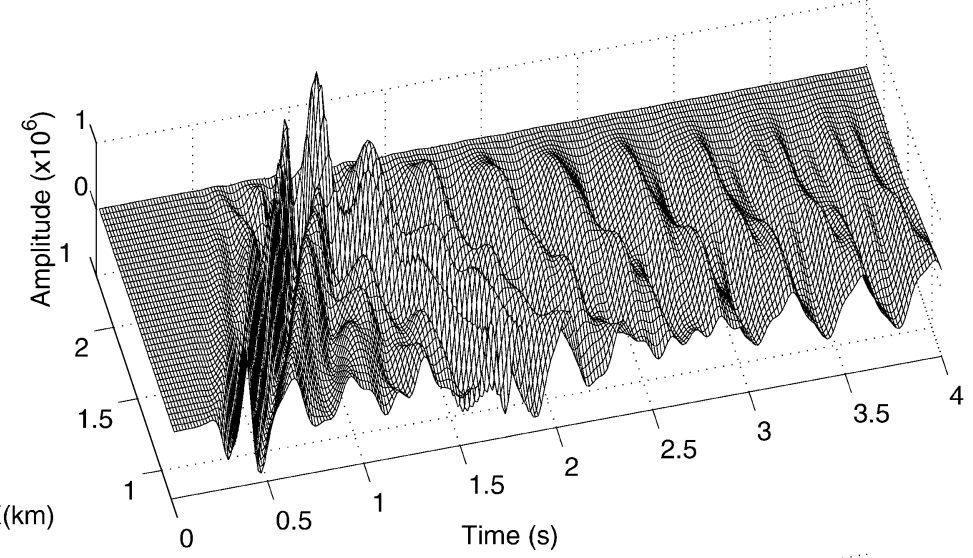

(c)

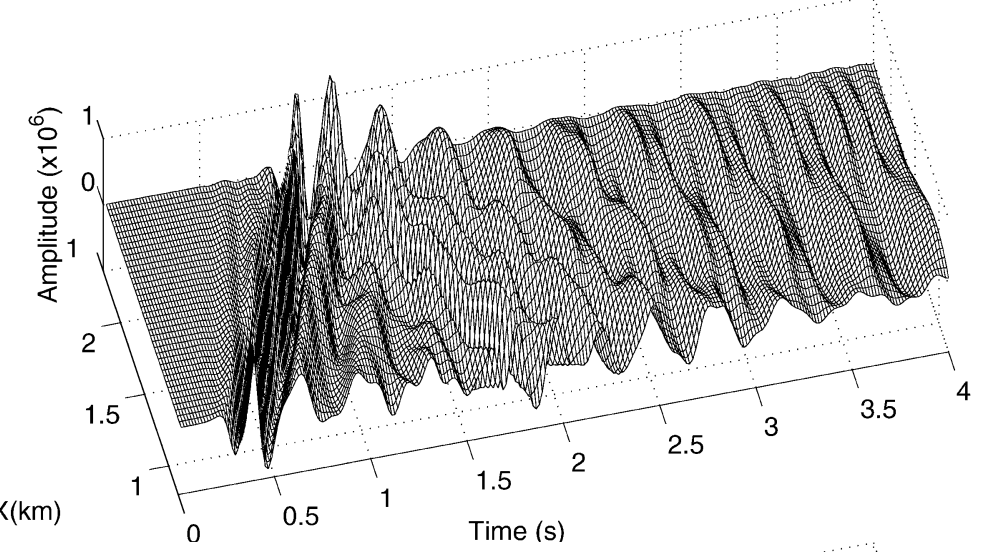

(d)

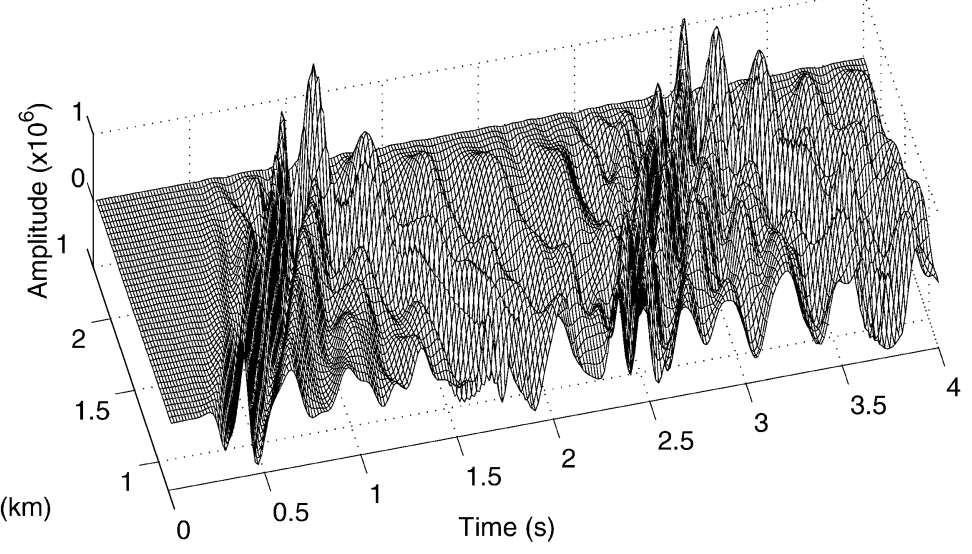

Fig. 6. Vertical displacements when the vertical load $\left(k_{z}=0\right)$ is placed in an elastic formation $400 \mathrm{~m}$ thick: (a) Model 1; (b) Model $2\left(\theta=45^{\circ}\right)$; (c) Model 2 $\left(\theta=90^{\circ}\right)$; (d) Model $3\left(\theta=45^{\circ}\right)$. 
(a)

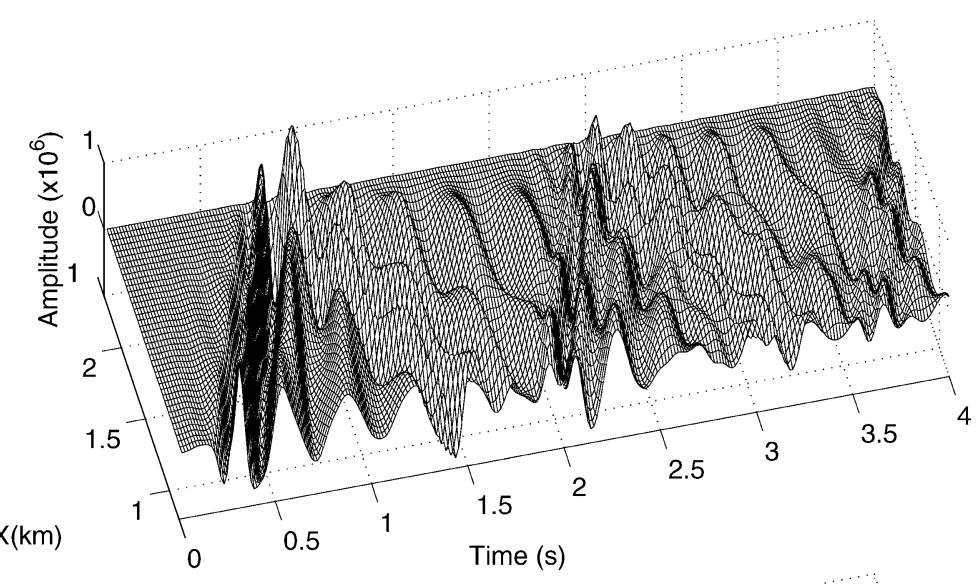

(b)

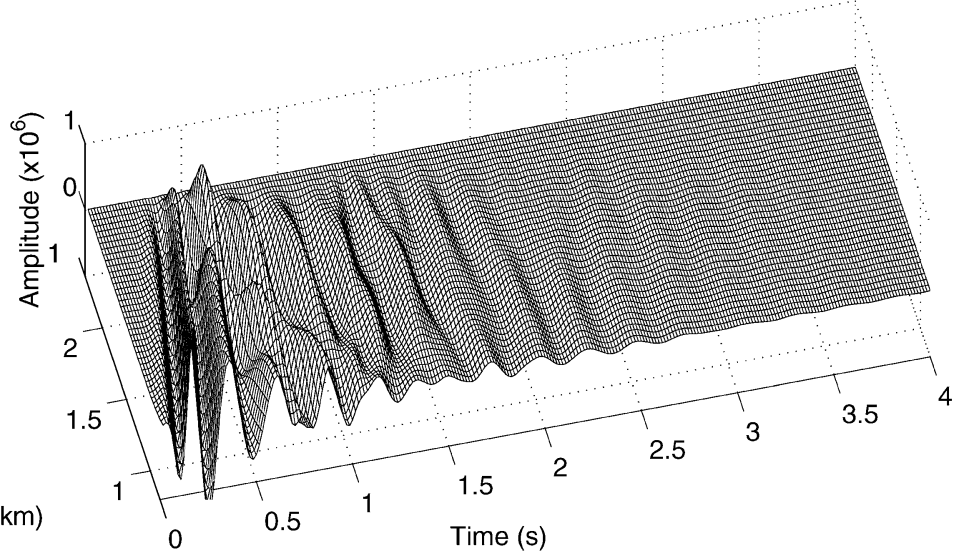

Fig. 7. Vertical displacements at receivers placed in Model 3, when the solid elastic formation is $400.0 \mathrm{~m}$ thick and $\theta=45^{\circ}$ for different apparent velocities: (a) $c=4208 \mathrm{~m} / \mathrm{s}$; (b) $c=2656 \mathrm{~m} / \mathrm{s}$.

The finite lateral extent of the valley surface generates additional surface waves at the edges, and resonance in a lateral direction. These effects tend to increase both the amplitude and the duration of the ground motion signatures.

As the thickness of the solid elastic formation decreases, the amplitude of the wavefield increases and additional guided surface waves appear. The decrease of the apparent wave velocity along the axis of the valley allowed the different wave types to be distinguished, particularly those associated with the slower velocities.

Appendix A. The Green's function for a 2.5D full-space

$$
\begin{aligned}
G_{x x}^{\text {full }} & =\frac{\mathrm{i}}{4 \rho \omega^{2}}\left[k_{\mathrm{s}}^{2} H_{0 \beta}-\frac{1}{r} B_{1}+\left(\frac{x-x_{0}}{r}\right)^{2} B_{2}\right] \\
G_{y y}^{\text {full }} & =\frac{\mathrm{i}}{4 \rho \omega^{2}}\left[k_{s}^{2} H_{0 \beta}-\frac{1}{r} B_{1}+\left(\frac{y-y_{0}}{r}\right)^{2} B_{2}\right] \\
G_{z z}^{\text {full }} & =\frac{\mathrm{i}}{4 \rho \omega^{2}}\left[k_{s}^{2} H_{0 \beta}-k_{z}^{2} B_{0}\right]
\end{aligned}
$$

$$
\begin{aligned}
G_{x y}^{\mathrm{full}} & =G_{y x}^{\mathrm{full}}=\frac{\mathrm{i}}{4 \rho \omega^{2}}\left(\frac{x-x_{0}}{r}\right)\left(\frac{y-y_{0}}{r}\right) B_{2} \\
G_{x z}^{\mathrm{full}} & =G_{z x}^{\mathrm{full}}=\frac{-k_{z}}{4 \rho \omega^{2}}\left(\frac{x-x_{0}}{r}\right) B_{1} \\
G_{y z}^{\mathrm{full}} & =G_{z y}^{\mathrm{full}}=\frac{-k_{z}}{4 \rho \omega^{2}}\left(\frac{y-y_{0}}{r}\right) B_{1}
\end{aligned}
$$

with

$r=\sqrt{\left(x-x_{0}\right)^{2}+\left(y-y_{0}\right)^{2}}, \quad H_{n \alpha}=H_{n}^{(2)}\left(k_{\alpha} r\right)$,

$H_{n \beta}=H_{n}^{(2)}\left(k_{\beta} r\right), \quad B_{n}=k_{\beta}^{n} H_{n \beta}-k_{\alpha}^{n} H_{n \alpha}$

in which the $H_{n}^{(2)}(\ldots)$ are second Hankel functions of order $n$.

\section{Appendix B. Definition of the Green's functions for a free-rigid layer}

This appendix lists the system of equations required to define the amplitude of the surface potentials. 
Load acting in the direction of the $x$-axis:

$\left[a_{i j}^{x} i=1, \ldots, 6 ; j=1, \ldots, 6\right]$

$\left[c_{i}^{x} i=1, \ldots, 6\right]=\left[b_{i}^{x} i=1, \ldots, 6\right]$

$a_{i j}^{x} ; i=1, \ldots, 6 ; j=1, \ldots, 6$

$a_{11}^{x}=-2 k_{n}^{2} \quad a_{12}^{x}=-k_{z}^{2} \quad a_{13}^{x}=k_{n}^{2}-\gamma_{n}^{2}$

$a_{14}^{x}=-a_{11}^{x} E_{b h} \quad a_{15}^{x}=-a_{12}^{x} E_{c h} \quad a_{16}^{x}=-a_{13}^{x} E_{c h}$

$a_{21}^{x}=-2 \quad a_{22}^{x}=1 \quad a_{23}^{x}=1$

$a_{24}^{x}=-a_{21}^{x} E_{b h} \quad a_{25}^{x}=-a_{22}^{x} E_{c h} \quad a_{26}^{x}=-a_{23}^{x} E_{c h}$

$a_{31}^{x}=\frac{-k_{s}^{2}}{\nu_{n}}-\frac{2 \nu_{z n}^{2}}{\nu_{n}} \quad a_{32}^{x}=0 \quad a_{33}^{x}=2 \gamma_{n}$

$a_{34}^{x}=a_{31}^{x} E_{b h} \quad a_{35}^{x}=0 \quad a_{36}^{x}=a_{33}^{x} E_{c h}$

$a_{41}^{x}=\frac{k_{n}^{2}}{\nu_{n}} E_{b h} \quad a_{42}^{x}=\frac{-k_{z}^{2}}{\gamma_{n}} E_{c h} \quad a_{43}^{x}=-\gamma_{n} E_{c h}$

$a_{44}^{x}=\frac{-k_{n}^{2}}{\nu_{n}} \quad a_{45}^{x}=\frac{-k_{z}^{2}}{\gamma_{n}} \quad a_{46}^{x}=-\gamma_{n}$

$a_{51}^{x}=-1 E_{b h} \quad a_{52}^{x}=0 \quad a_{53}^{x}=1 E_{c h}$

$a_{54}^{x}=1 \quad a_{55}^{x}=0 \quad a_{56}^{x}=-1$

$a_{61}^{x}=\frac{-1}{\nu_{n}} E_{b h} \quad a_{62}^{x}=\frac{1}{\gamma_{n}} E_{c h} \quad a_{63}^{x}=0$

$a_{64}^{x}=\frac{-1}{\nu_{n}} \quad a_{65}^{x}=\frac{1}{\gamma_{n}} \quad a_{66}^{x}=0$

$c_{i}^{x} i=1, \ldots, 6$

$c_{1}^{x}=A_{n}^{x} \quad c_{2}^{x}=B_{n}^{x} \quad c_{3}^{x}=C_{n}^{x}$

$c_{4}^{x}=D_{n}^{x} \quad c_{5}^{x}=E_{n}^{x} \quad c_{6}^{x}=F_{n}^{x}$

$b_{i}^{x} i=1, \ldots, 6$

$b_{1}^{x}=-2 k_{n}^{2} E_{b 1}+\left(-k_{s}^{2}+2 k_{n}^{2}\right) E_{c 1} \quad b_{2}^{x}=-2 E_{b 1}+2 E_{c 1}$

$b_{3}^{x}=\left(\frac{k_{s}^{2}}{\nu_{n}}+\frac{2 \nu_{z n}^{2}}{\nu_{n}}\right) E_{b 1}-2 \gamma_{n} E_{c 1}$

$b_{4}^{x}=\frac{k_{n}^{2}}{\nu_{n}} E_{b h 1}+\left(\gamma_{n}+\frac{k_{z}^{2}}{\gamma_{n}}\right) E_{c h 1}$

$b_{5}^{x}=1 E_{b h 1}-1 E_{c h 1} \quad b_{6}^{x}=\frac{1}{\nu_{n}} E_{b h 1}-\frac{1}{\gamma_{n}} E_{c h 1}$

with

$$
\begin{aligned}
& E_{b 1}=\mathrm{e}^{-\mathrm{i} \nu_{n} y_{0}}, \quad E_{c 1}=\mathrm{e}^{-\mathrm{i} \gamma_{n} y_{0}}, \quad \nu_{z n}=\sqrt{-k_{z}^{2}-k_{n}^{2}}, \\
& E_{b h}=\mathrm{e}^{-\mathrm{i} \nu_{n} h}, \quad E_{c h}=\mathrm{e}^{-\mathrm{i} \gamma_{n} h}, \quad E_{b h 1}=\mathrm{e}^{-\mathrm{i} \nu_{n}\left|h-y_{0}\right|}, \\
& E_{c h 1}=\mathrm{e}^{-\mathrm{i} \gamma_{n}\left|h-y_{0}\right|} .
\end{aligned}
$$

Load acting in the direction of the $y$-axis:

$\left[a_{i j}^{y} i=1, \ldots, 6 ; j=1, \ldots, 6\right]$

$\left[c_{i}^{y} i=1, \ldots, 6\right]=\left[b_{i}^{y} i=1, \ldots, 6\right]$

$a_{i j}^{y} i=1, \ldots, 6 ; j=1, \ldots, 6$

$a_{11}^{y}=-2 \nu_{n} \quad a_{12}^{y}=\frac{-k_{n}^{2}}{\gamma_{n}}+\gamma_{n} \quad a_{13}^{y}=\frac{-k_{z}^{2}}{\gamma_{n}}$

$a_{14}^{y}=a_{11}^{y} E_{b h} \quad a_{15}^{y}=a_{12}^{y} E_{c h} \quad a_{16}^{y}=a_{13}^{y} E_{c h}$

$a_{21}^{y}=-2 \nu_{n} \quad a_{22}^{y}=\frac{-k_{n}^{2}}{\gamma_{n}} \quad a_{23}^{y}=\frac{-k_{z}^{2}}{\gamma_{n}}+\gamma_{n}$

$a_{24}^{y}=a_{21}^{y} E_{b h} \quad a_{25}^{y}=a_{22}^{y} E_{c h} \quad a_{26}^{y}=a_{23}^{y} E_{c h}$

$a_{31}^{y}=-k_{s}^{2}-2 \nu_{z n}^{2} \quad a_{32}^{y}=-2 k_{n}^{2} \quad a_{33}^{y}=-2 k_{z}^{2}$

$a_{34}^{y}=-a_{31}^{y} E_{b h} \quad a_{35}^{y}=-a_{32}^{y} E_{c h} \quad a_{36}^{y}=-a_{33}^{y} E_{c h}$

$a_{41}^{y}=-1 E_{b h} \quad a_{42}^{y}=1 E_{c h} \quad a_{43}^{y}=0$

$a_{44}^{y}=1 \quad a_{45}^{y}=-1 \quad a_{46}^{y}=0$

$a_{51}^{y}=-\nu_{n} E_{b h} \quad a_{52}^{y}=\frac{-k_{n}^{2}}{\gamma_{n}} E_{c h} \quad a_{53}^{y}=\frac{-k_{z}^{2}}{\gamma_{n}} E_{c h}$

$a_{54}^{y}=-\nu_{n} \quad a_{55}^{y}=\frac{-k_{n}^{2}}{\gamma_{n}} \quad a_{56}^{y}=\frac{-k_{z}^{2}}{\gamma_{n}}$

$a_{61}^{y}=-1 E_{b h} \quad a_{62}^{y}=0 \quad a_{63}^{y}=1 E_{c h}$

$a_{64}^{y}=1 \quad a_{65}^{y}=0 \quad a_{66}^{y}=-1$

$c_{i}^{y} \quad i=1, \ldots, 6$

$c_{1}^{y}=A_{n}^{y} \quad c_{2}^{y}=B_{n}^{y} \quad c_{3}^{y}=C_{n}^{y}$

$c_{4}^{y}=D_{n}^{y} \quad c_{5}^{y}=E_{n}^{y} \quad c_{6}^{y}=F_{n}^{y}$

$b_{i}^{y} \quad i=1, \ldots, 6$

$b_{1}^{y}=2 \nu_{n} E_{b 1}-\left(\frac{\nu_{z n}^{2}}{\gamma_{n}}+\gamma_{n}\right) E_{c 1}$

$b_{2}^{y}=2 \nu_{n} E_{b 1}-\left(\frac{\nu_{z n}^{2}}{\gamma_{n}}+\gamma_{n}\right) E_{c 1}$

$b_{3}^{y}=\left(-k_{s}^{2}-2 \nu_{z n}^{2}\right) E_{b 1}+2 \nu_{z n}^{2} E_{c 1} \quad b_{4}^{y}=1 E_{b h 1}-1 E_{c h 1}$

$b_{5}^{y}=\nu_{n} E_{b h 1}-\frac{\nu_{z n}^{2}}{\gamma_{n}} E_{c h 1} \quad b_{6}^{y}=1 E_{b h 1}-1 E_{c h 1}$

with
$E_{b 1}=\mathrm{e}^{-\mathrm{i} \nu_{n} y_{0}}$
$E_{c 1}=\mathrm{e}^{-\mathrm{i} \gamma_{n} y_{0}}$,
$\nu_{z n}=\sqrt{-k_{z}^{2}-k_{n}^{2}}$,
$E_{b h}=\mathrm{e}^{-\mathrm{i} \nu_{n} h}$
$E_{c h}=\mathrm{e}^{-\mathrm{i} \gamma_{n} h}$,
$E_{b h 1}=\mathrm{e}^{-\mathrm{i} \nu_{n}\left|h-y_{0}\right|}$,
$E_{c h 1}=\mathrm{e}^{-\mathrm{i} \gamma_{n}\left|h-y_{0}\right|}$. 
Load acting in the direction of the $z$-axis:

$$
\begin{aligned}
& {\left[a_{i j}^{z} i=1, \ldots, 6 ; j=1, \ldots, 6\right]} \\
& {\left[c_{i}^{z} i=1, \ldots, 6\right]=\left[b_{i}^{z} i=1, \ldots, 6\right]} \\
& a_{i j}^{z} \quad i=1, \ldots, 6 ; \quad j=1, \ldots, 6 \\
& a_{11}^{z}=-2 \quad a_{12}^{z}=1 \quad a_{13}^{z}=1 \\
& a_{14}^{z}=-a_{11}^{z} E_{b h} \quad a_{15}^{z}=-a_{12}^{z} E_{c h} \quad a_{16}^{z}=-a_{13}^{z} E_{c h} \\
& a_{21}^{z}=-2 k_{z}^{2} \quad a_{22}^{z}=k_{z}^{2}-\gamma_{n}^{2} \quad a_{23}^{z}=-k_{n}^{2} \\
& a_{24}^{z}=-a_{21}^{z} E_{b h} \quad a_{25}^{z}=-a_{22}^{z} E_{c h} \quad a_{26}^{z}=-a_{23}^{z} E_{c h} \\
& a_{31}^{z}=-\left(\frac{k_{s}^{2}}{\nu_{n}}+\frac{2 \nu_{z n}^{2}}{\nu_{n}}\right) \quad a_{32}^{z}=2 \gamma_{n} \quad a_{33}^{z}=0 \\
& a_{34}^{z}=a_{31}^{z} E_{b h} \quad a_{35}^{z}=a_{32}^{z} E_{c h} \quad a_{36}^{z}=0 \\
& a_{41}^{z}=\frac{-1}{\nu_{n}} E_{b h} \quad a_{42}^{z}=0 \quad a_{43}^{z}=\frac{1}{\gamma_{n}} E_{c h} \\
& a_{44}^{z}=\frac{-1}{\nu_{n}} \quad a_{45}^{z}=0 \quad a_{46}^{z}=\frac{1}{\gamma_{n}} \\
& a_{51}^{z}=-1 E_{b h} \quad a_{52}^{z}=1 E_{c h} \quad a_{53}^{z}=0 \\
& a_{54}^{z}=1 \quad a_{55}^{z}=-1 \quad a_{56}^{z}=0 \\
& a_{61}^{z}=\frac{-k_{z}^{2}}{\nu_{n}} E_{b h} \quad a_{62}^{z}=-\gamma_{n} E_{c h} \quad a_{63}^{z}=\frac{-k_{n}^{2}}{\gamma_{n}} E_{c h} \\
& a_{64}^{z}=\frac{-k_{x}^{2}}{\nu_{n}} \quad a_{65}^{z}=-\gamma_{n} \quad a_{66}^{z}=\frac{-k_{n}^{2}}{\gamma_{n}} \\
& c_{i}^{z} \quad i=1, \ldots, 6 \\
& c_{1}^{z}=A_{n}^{z} \quad c_{2}^{z}=B_{n}^{z} \quad c_{3}^{z}=C_{n}^{z} \\
& c_{4}^{z}=D_{n}^{z} \quad c_{5}^{z}=E_{n}^{z} \quad c_{6}^{z}=F_{n}^{z} \\
& b_{i}^{z} \quad i=1, \ldots, 6 \\
& b_{1}^{z}=2\left(-E_{b 1}+E_{c 1}\right) \\
& b_{2}^{z}=-2 k_{z}^{2} E_{b 1}+\left(k_{z}^{2}-\gamma_{n}^{2}-k_{n}^{2}\right) E_{c 1} \\
& b_{3}^{z}=\left(\frac{k_{s}^{2}}{\nu_{n}}+\frac{2 \nu_{z n}^{2}}{\nu_{n}}\right) E_{b 1}-2 \gamma_{n} E_{c 1} \\
& b_{4}^{z}=\frac{1}{\nu_{n}} E_{b h 1}-\frac{1}{\gamma_{n}} E_{c h 1} \\
& b_{5}^{z}=1 E_{b h 1}-1 E_{c h 1} \quad b_{6}^{z}=\frac{k_{z}^{2}}{\nu_{n}} E_{b h 1}+\left(\frac{k_{n}^{2}}{\nu_{n}}+\gamma_{n}\right) E_{c h 1}
\end{aligned}
$$
motion. Bull Seismol Soc Am 1973;63:283-98.

[2] Sánchez-Sesma FJ. Site effects on strong ground motion. Soil Dyn Earthquake Engng 1987;6:124-32.

[3] Aki K. Local site effect on ground motion. In: Von Thun JL, editor. Earthquake engineering and soil dynamics II: recent advances in ground-motion evaluation. ASCE; 1988. p. 103-55.

[4] Sánchez-Sesma FJ. Diffraction of elastic waves by three-dimensional surface irregularities. Bull Seismol Soc Am 1983;73:1621-36.

[5] Moeen-Vaziri N, Trifunac MD. Scattering and diffraction of plane $P$ and SV waves by two-dimensional inhomogeneities: Part II. Soil Dyn Earthquake Engng 1988;7:189-200.

[6] Lee VW, Wu X. Application of the weighted residual method to diffraction by 2-D canyons of arbitrary shape: II. Incident, P, SV, and Rayleigh waves. Soil Dyn Earthquake Engng 1994;13:365-75.

[7] Todorovska MI, Hayir A, Trifunac MD. Antiplane response of a dike on flexible embedded foundation to incident SH-waves. Soil Dyn Earthquake Engng 2001;21(7):593-601.

[8] Hayir A, Todorovska MI, Trifunac MD. Antiplane response of a dike with flexible soil-structure interface to incident SH-waves. Soil Dyn Earthquake Engng 2001;21(7):603-13.

[9] Kausel E. Forced vibrations of circular foundations in layered media. MIT Research Report 70-3. Cambridge. MA: Department of Civil Engineering, Massachusetts Institute of Technology; 1974.

[10] Zhang L, Chopra AK. Three dimensional analyses of spatially varying ground motions around a uniform canyon in a homogeneous halfspace. Int J Earthquake Engng Struct Dyn 1991;20:911-26.

[11] Sánchez-Sesma FJ, Rosenblueth E. Ground motion on alluvial valleys under incident plane SH waves. Int J Earthquake Engng Struct Dyn 1979;7:441-50.

[12] Wong HL. Effect of surface topography on the diffraction of P, SV, and Rayleigh waves. Bull Seismol Soc Am 1982;72:1167-83.

[13] Sánchez-Sesma FJ, Campillo N. Diffraction of P, SV, and Rayleigh waves by topographical features: a boundary integral formulation. Bull Seismol Soc Am 1991;81:2234-53.

[14] Sánchez-Sesma FJ, Campillo N. Topographical effects for incident $P$, SV, and Rayleigh waves. Tectonophysics 1993;218:113-25.

[15] Bouchon M. A simple, complete numerical solution to the problem of diffraction of SH waves by an irregular surface. J Acoust Soc Am 1985;77:1-5.

[16] Kawase H. Time-domain response of a semicircular canyon for incident SV, $\mathrm{P}$ and Rayleigh waves calculated by the discrete wavenumber boundary element method. Bull Seismol Soc Am 1988; 78:1415-37.

[17] Ohtsuki A, Harumi K. Effect of topography and subsurface inhomogeneities on seismic SV waves. Int J Earthquake Engng Struct Dyn 1983;11:441-62.

[18] Shah AH, Wong KC, Datta SK. Diffraction of plane SH waves in a half-space. Int J Earthquake Engng Struct Dyn 1982;10:519-28.

[19] Sánchez-Sesma FJ, Pérez-Rocha LE, Chávez-Pérez S. Diffraction of elastic waves by three-dimensional surface irregularities: Part II. Bull Seismol Soc Am 1989;79:101-12.

[20] Frankel A, Vidale J. A three-dimensional simulation of seismic waves in the Santa Clara Valley, California, from a Loma Prieta aftershock. Bull Seismol Soc Am 1992;82:2045-74.

[21] Frankel A. Three-dimensional simulations of ground motions in the San Bernardino Valley, California, for hypothetical earthquakes on the San Andreas fault. Bull Seismol Soc Am 1993;83:1020-41.

[22] Kim J, Papageorgiou AS. Discrete wavenumber boundary element method for 3-D scattering problems. J Engng Mech ASCE 1993; 119(3):603-24.

[23] Reinoso E, Wrobel LC, Power H. Three-dimensional scattering of seismic waves from topographical structures. Soil Dyn Earthquake Engng 1997;16:41-61. 
[24] Kattis SE, Polyzos D, Beskos DE. Modelling of pile wave barriers by effective trenches and their screening effectiveness. Soil Dyn Earthquake Engng 1999;18(1):1-10.

[25] Bard P-Y, Bouchon M. The two-dimensional resonance of sedimentfilled valleys. Bull Seismol Soc Am 1985;75:519-41.

[26] Zhou T, Dravinski M. Resonance prediction of deep sediment valleys through an eigenvalue method. Geophys J Int 1984;117: $749-62$.

[27] Luco JE, Long HL, De Barros FCP. Three dimensional response of a cylindrical canyon in a layered half-space. Earthquake Engng Struct Dyn 1990;19(6):799-817.

[28] Luco JE, De Barros FCP. Three-dimensional response of a layered cylindrical valley embedded in a layered half-space. Earthquake Engng Struct Dyn 1995;24(1):109-25.

[29] Zhang L, Chopra AK. Three-dimensional analysis of spatially varying ground motions around a uniform canyon in a homogeneous half-space. Earthquake Engng Struct Dyn 1991;20(10): 911-26.

[30] Zhang L, Chopra AK. Impedance functions for three-dimensional foundations supported on an infinitely-long canyon of uniform crosssection in a homogeneous half-space. Earthquake Engng Struct Dyn 1991;20(11):1011-27.

[31] Pedersen HA, Sánchez-Sesma FJ, Campillo M. Three-dimensional scattering by two-dimensional topographies. Bull Seismol Soc Am 1994;84:1169-83.

[32] Luco JE, De Barros FCP. Seismic response of a cylindrical shell embedded in a layered viscoelastic half-space. I: formulation. Earthquake Engng Struct Dyn 1994;23(5):553-67.

[33] De Barros FCP, Luco JE. Seismic response of a cylindrical shell embedded in a layered viscoelastic half-space. II: validation and numerical results. Earthquake Engng Struct Dyn 1994;23(5):569-80.

[34] De Barros FCP, Luco JE. Response of a layered viscoelastic halfspace to a moving point load. Wave Motion 1994;19:189-210.

[35] Stamos AA, Beskos DE. 3-D seismic response analysis of long lined tunnels in half-space. Soil Dyn Earthquake Engng 1996;15:111-8.

[36] Santos P, António J, Tadeu A. Wave scattering by 2D smooth topographical elastic deformations caused by point blast source. J Comput Model Engng Sci, CMES 2000;1(4):79-98.

[37] Tadeu A, Santos P. 3D wave propagation in fluid-filled irregular boreholes in elastic formations. J Soil Dyn Earthquake Engng 2001; 21(6):499-517.

[38] Pei D, Papageorgiou AS. Locally generated surface waves in Santa Clara valley: analysis of observations and numerical simulation. Earthquake Engng Struct Dyn 1996;25(1):47-63.

[39] Huang H-C, Chiu H-C. Canyon topography effects on ground motion at Feitsui damsite. Soil Dyn Earthquake Engng 1999;18(2):87-99.

[40] Davis CA, Lee VW, Bardet JP. Transverse response of underground cavities and pipes to incident SV waves. Earthquake Engng Struct Dyn 2001;30:383-410.
[41] Rassem M, Heidebrecht AC, Ghobarah A. A simple engineering model for the seismic site response of alluvial valleys. Soil Dyn Earthquake Engng 1995;14(3):199-210.

[42] Rassem M, Ghobarah A, Heidebrecht AC. Engineering perspectives for the seismic site response of alluvial valleys. Earthquake Engng Struct Dyn 1997;26(4):477-93.

[43] Guan F, Moore ID, Spyrakos CC. Two dimensional transient fundamental solution due to suddenly applied load in a half-space. Soil Dyn Earthquake Engng 1998;17(4):269-77.

[44] Dineva PS, Manolis GD. Scattering of seismic waves by cracks in multi-layered geological regions. I. Mechanical model. Soil Dyn Earthquake Engng 2001;21(7):615-25.

[45] Dineva PS, Manolis GD. Scattering of seismic waves by cracks in multi-layered geological regions, II. Numerical results. Soil Dyn Earthquake Engng 2001;21(7):627-41.

[46] Tadeu A, António J. 2.5D Green's functions for elastodynamic problems in layered acoustic and elastic formations. J Comput Model Engng Sci, CMES 2001;2(4):477-95.

[47] Tadeu A, António J, Godinho L. Green's function for two-and-a-half dimensional elastodynamic problems in a half-space. Comput Mech 2001;27(6):484-91.

[48] Tadeu AJB, Kausel E. Green's functions for two-and-a-half dimensional elastodynamic problems. J Engng Mech, ASCE 2000;126(10): $1093-7$.

[49] Papageorgiou AS, Pei D. A discrete wavenumber boundary element method for study of 3-D response of 2-D scatterers. Earthquake Engng Struct Dyn 1998;27(6):619-38.

[50] Lamb H. On the propagation of tremors at the surface of an elastic solid. Philos Trans R Soc Lond 1904;A203:1-42.

[51] Bouchon M. Discrete wave number representation of elastic wave fields in three-space dimensions. J Geophys Res 1979;84:3609-14.

[52] Phinney RA. Theoretical calculation of the spectrum of first arrivals in layered elastic mediums. J Geophys Res 1965;70:5107-23.

[53] Bouchon M, Aki K. Time-domain transient elastodynamic analysis of 3D solids by BEM. Int J Numer Meth Engng 1977;26:1709-28.

[54] Beskos DE. Boundary element methods in dynamic analysis: Part II (1986-1996). Appl Mech Rev 1997;50(3):149-97.

[55] Manolis GD, Beskos DE. Boundary element methods in elastodynamics. London: Unwin Hyman; 1988.

[56] Tadeu AJB, Santos PFA, Kausel E. Closed-form integration of singular terms for constant, linear and quadratic boundary elementsPart I: SH wave propagation. Engng Anal Bound Elem 1999;23(8): 671-81.

[57] Tadeu AJB, Santos PFA, Kausel E. Closed-form integration of singular terms for constant, linear and quadratic boundary elementsPart II: SV-P wave propagation. Engng Anal Bound Elem 1999; 23(9):757-68.

[58] Kausel E, Roesset JM. Frequency domain analysis of undamped systems. J Engng Mech ASCE 1992;118(4):721-34. 